\title{
Applying Encryption Algorithm Practically On M-commerce Background: Observing Security and Crucial Performance
}

\author{
Trivedi Jaydipkumar $\mathrm{H}^{1}$ Patel Jagdishkumar $\mathrm{M}^{2}$ Trivedi Harakh $\mathrm{J}^{3}$ \\ Kuldip Dhirajbhai Pipaliya ${ }^{4}$ \\ ${ }^{I}$ Assistant Professor MCMSR College, Visnagar. ${ }^{2}$ Assistant Professor MCMSR College, Visnagar. \\ ${ }^{3}$ Lt.Scientist, Vikram A Sarabhai Community Science Center. Ahmedabad ${ }^{4}$ SVIPL PVT LTD
}

\begin{abstract}
The encryption algorithm is applying on M-commerce background and consequently observing security and performance. The work is observing website based performance as well as wireless toolkit 2.5, emulator based performance. The customers are establishing their $\mathrm{m}$-commerce transaction on broadcasting agents based m-commerce background. For achieving system side as well as customer side security both the participant need encryption algorithm. The work shows the curtail role of the performance of the encryption algorithm practically on M-commerce background. The system is using customer credit conformation system. And if the customer found authentic he will be allowed for doing transaction. The work also indicates and explains how the algorithm is functioning.
\end{abstract}

Keywords: - M-Commerce.

\section{INTRODUCTION}

Encryption algorithm is using for security purpose as well as it is stimulating efficient m-commerce transaction. The customer is establishing his $\mathrm{m}$-commerce transaction on broadcasting agents based back ground. ${ }^{[1]}$ Any would be infiltrator is using the normal system facilities to access the database. The work is instructing the applications of encryption algorithm on m-commerce background. Encryption algorithm is stand for encrypting the data and storing the data into database in encrypted style. The work is suggesting the use of encryption algorithm and showing the practical approach in the application. ${ }^{[2]}$

\section{LITERATURE REVIEW}

Trivedi J H, Darji J H, Patel H D, Trivedi P H, have depicted designing and prototyping encryption algorithm: Working as secure M-commerce transaction of broadcasting and its receiving agent based $\mathrm{m}$ commerce model. The work has explored the study of encryption algorithm and considering the user name of the e-mail address as the secure encryption key. The work has explored the real designing of encryption algorithm and indicated secure transaction. "M-payment between banks using sms" have depicted by Soni P, written about payment systems and sms played important role.

(1) Study the encryption algorithm.

\section{OBJECTIVES}

(2) Applying encryption algorithm practically on M-commerce background.

(3) Observing security and crucial performance as result of encryption algorithm

(4) Study the m-commerce model.

\section{RESEARCH METHODOLOGY}

Research Methodology used in this research paper is purely excremental.

\section{HYPOTHESIS}

-There is security on m-commerce background if we are using encryption algorithm.

\section{ENCRYPTION ALGORITHM}

Encryption algorithm has been used for encrypting the text ${ }^{[2]}$ Encrypted text is to be stored in to database. The encrypted text is in not understandable format so it very secure from user as well as System administrator. Present work proves that the encryption algorithm is initiating the M-commerce transaction.

Step 1: TRIVEDI-PRATIKSHA-J

(Plain text as message obtained, converts the character to its related alphabetical digit like $\mathrm{A}=01, \mathrm{~B}=02$, $\mathrm{C}=03, \mathrm{D}=04$.In the plain text blanks seems as “-“. Indicates Space as 00) 
Step 2: 20180922050409001618012009111908010010

(Decide the digital block of the string as per the length of user name of e-mail address's digit)

Step 3: Pbpathak1978(Got from mrjlecturer@yahoo.com.

The encryption key formed on the basis of user name of e-mail address taken form e-mail message for business Transaction is taking user name of e-mail address only without "@” and rest of the address like yahoo mail.com)

16021601090801111978 (Encryption key got from username,

Converts the character to its related alphabetical Digit like $\mathrm{A}=01, \mathrm{~B}=02, \mathrm{C}=03, \mathrm{D}=04 \ldots .$.

Step 4: 20180922050409001618012009111908010010 $\underline{16021601090801111978} \underline{160216010908011119}$ 36202523141210113596172225122816021129

( Sum the digital block of the string with Encryption key)

Step 5: CFBOBEBCADABA0AACEIF AGBBBEABBHAF0BAABI

( The obtained result of the sum from step 4 converted digital string into alphabetical String like 3=C, 9=I, and keep Zero 0 remain Same for further task, It means 0 is encrypted as 0.

\section{Encrypted Message:}

\section{CFBOBEBCADABA0AACEIF AGBBBEABBHAF0BAABI}

\section{Step 6: CFBOBEBCADABA0AACEIF AGBBBEABBHAFOBAABI} 36202523141210113596172225122816021129

( Converted the alphabetical string into digital String like C=3, I=9, and keep Zero "0" remain Same for further task, It means " 0 " is encrypted as " 0 " only.)

Step 7: 36202523141210113596172225122816021129

$\underline{16021601090801111978} \underline{160216010908011119}$

20180922050409001618012009111908010010

(Subtracted the digital block of string with Encryption key)

Step 8: 20180922050409001618012009111908010010

T R I VE D I - PR A T IK S H A - J

(Alphabetical string on the basis of result acquired from subtraction)

The under mention screen shot of Simulator of Wireless Toolkit 2.5 with Java Code on which the encrypting algorithm is performing practically.

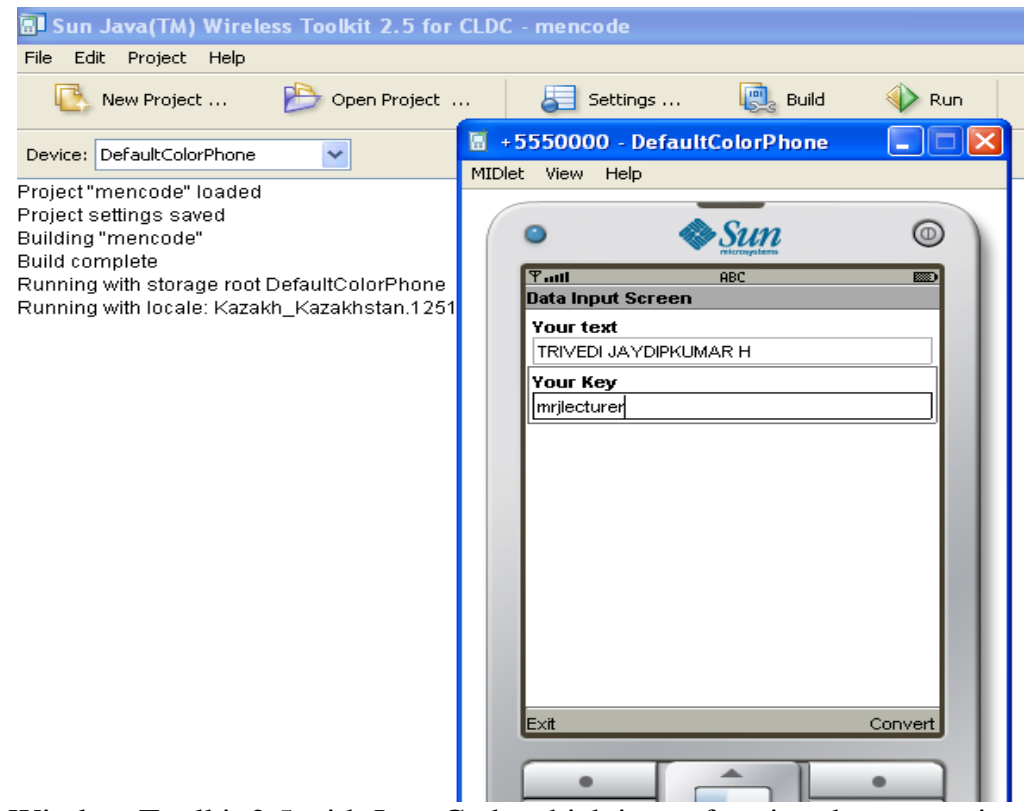

Figure 1: Wireless Toolkit 2.5 with Java Code which is performing the encrypting algorithm 
/** To change this license header, choose License Headers in Project Properties.

* To change this template file, choose Tools | Templates

$/ / *$ and open the template in the editor.

$* /$

//package encoder;

import javax.microedition.lcdui.*;

import javax.microedition.midlet.*;

/**

$* \mathrm{Pt}$

$* /$

public class mencode extends MIDlet implements

CommandListener\{ private Display display;

private Form inputForm, convertedForm,

encodedForm, resultForm;

private TextField iT1, iT2, cT1, cT2, eT1, eT2,

rT1, rT2;

private Command $\mathrm{iC}, \mathrm{cC}, \mathrm{eC}, \mathrm{rC}$, exit;

public mencode ()\{

initInputForm(); display = Display.getDisplay $($ this $) ;\}$

public void initInputForm() \{ inputForm = new Form("Data Input Screen"); iT1 = new TextField("Your text", null, 255,

TextField.ANY); iT2 = new TextField("Your Key", null, 255,

TextField.ANY); inputForm.append(iT1); inputForm.append(iT2);

$\mathrm{iC}=$ new Command("Convert",

Command.ITEM, 1)

exit $=$ new Command("Exit", Command.EXIT,

2);

inputForm.addCommand(iC);

inputForm.addCommand(exit);

inputForm.setCommandListener(this); \}

public void initConvertedForm() \{

convertedForm = new Form $($ "Converted Data Screen");

cT1 = new TextField("Your text", null, 255,

TextField.ANY);

cT2 = new TextField("Your Key", null, 255,

TextField.ANY);

convertedForm.append(cT1);

convertedForm.append(cT2)

$\mathrm{cC}=$ new Command("Encoded",

Command.ITEM, 1);

exit $=$ new Command ("Exit", Command.EXIT,

2);

convertedForm.addCommand(cC);

convertedForm.addCommand(exit);

convertedForm.setCommandListener(this); \}

public void initEncodedForm() \{

encodedForm = new Form ("Encoded Data

Screen");

eT1 = new TextField("Your text", null, 255,

TextField.ANY);

eT2 = new TextField("Your Key", null, 255,

TextField.ANY);

encodedForm.append(eT1);

encodedForm.append(eT2);

$\mathrm{eC}=$ new Command("Result", Command.ITEM,

1)

exit $=$ new Command("Exit", Command.EXIT,

2);

encodedForm.addCommand(eC);

encodedForm.addCommand(exit);

encodedForm.setCommandListener(this);

\} public void initResultForm() \{

resultForm = new Form("Result Data Screen");

rT1 = new TextField("Your text", null, 255,

TextField.ANY);

rT2 = new TextField("Your Key", null, 255,

TextField.ANY);

resultForm.append(rT1);

resultForm.append(rT2);

1)

$\mathrm{rC}=$ new Command("Home", Command.ITEM,

exit = new Command("Exit", Command.EXIT,

2);

resultForm.addCommand(rC);

resultForm.addCommand(exit);

resultForm.setCommandListener(this); \}

public void startApp ()\{

display.setCurrent(inputForm); \}

public void pause $A p p()\{$

public void destroyApp(boolean unconditional)

protected void convertData() \{

char[] orgText;

String convertedString = "";

int asciiValue;

orgText $=\mathrm{iT} 1$.getString ()$\cdot \operatorname{toCharArray}()$;

for (int $\mathrm{i}=0$; $\mathrm{i}<$ orgText.length; $\mathrm{i}++$ ) \{

asciiValue $=($ int $)$ orgText $[\mathrm{i}]$;

if (asciiValue $>=65 \& \&$ asciiValue $<=90)\{$ asciiValue $=$ asciiValue -64 ; if (asciiValue <=9) \{ // if substraction is

less than 10 than ' 0 ' is append to that sub convertedString $=$ convertedString + " $0 "$

+ asciiValue;

+ asciiValue \} else $\{$ convertedString $=$ convertedString

\section{\}
FOR SYMBOLES}

if (asciiValue >=97 \&\& asciiValue $<=122$ )

asciiValue $=$ asciiValue -96 ;

if (asciiValue $<=9$ ) 

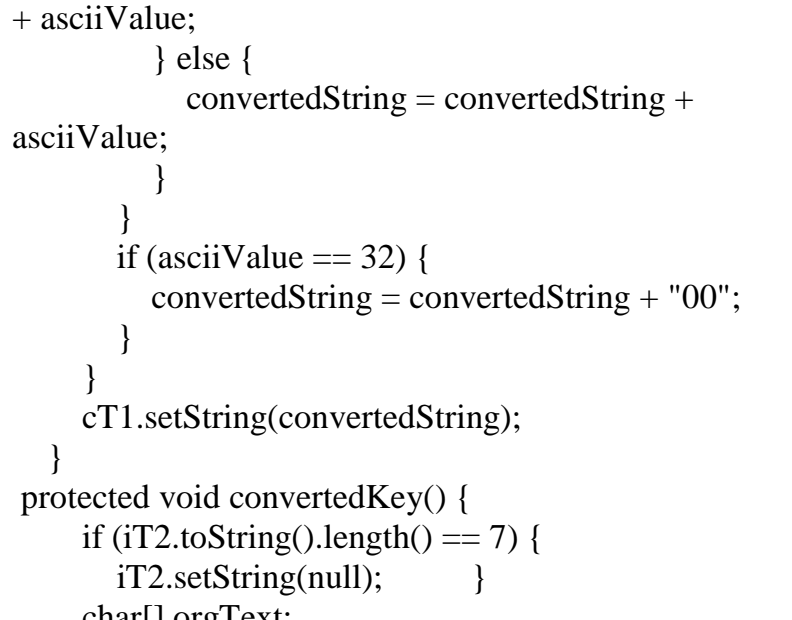

partData $=$
Integer.parseInt(data.substring(dataStrTrack,

dataStrTrack + 2));

partKey $=$

Integer.parseInt(key.substring(keyStrTrack,

keyStrTrack + 2));

resultData = partData + partKey;

tmpStr = String.valueOf(resultData);

if (resultData $<=9)$ \{

tmpStr $=$ "0" + tmpStr; $\}$

// encodedForm.append("ไn" + partData + "+"

+ partKey + "=" + tmpStr);

resultStr $+=$ tmpStr;

if (flag) \{

break; \}

keyStrTrack $=$ keyStrTrack +2 ;

dataStrTrack $=$ dataStrTrack +2 ;

if $($ keyStrTrack $>=$ keyLen/*6*/) \{ keyStrTrack $=0 ; \quad\} \quad\}$

eT1.setString(resultStr); \}

protected void decodeData() \{

int dataLen $=\mathrm{eT} 1$.getString().length();

int keyLen = cT2.getString().length();

String data $=\mathrm{eT} 1$.getString () ;

String key $=$ cT2 .getString();

int partData $=0$, partKey $=0$, dataStrTrack $=0$,

convertedString $=$ convertedString + "0" + asciiValue;

\} else $\{$ convertedString $=$ convertedString + asciiValue; \} \}

if (asciiValue $>=97 \& \&$ asciiValue $<=122)\{$ asciiValue $=$ asciiValue -96 ;

if (asciiValue <=9) \{

convertedString $=$ convertedString $+" 0 "+$ asciiValue;

\} else $\{$ convertedString $=$ convertedString + asciiValue; \} \}

if $($ asciiValue $==32)\{$

\} \}

convertedString = convertedString + "00";

cT2.setString(convertedString); \}

protected void encodedData() \{

int dataLen $=$ cT1 .getString $($ ).length $($ );

int keyLen $=\mathrm{cT} 2$.getString().length();

String data $=$ cT1.getString () ;

String key $=$ cT2.getString () ;

int partData $=0$, partKey $=0$, dataStrTrack $=0$,

keyStrTrack $=0$, resultData;

String resultStr = "", tmpStr = "";

boolean flag = false;

// encodedForm.append("InDATA:-" + data);

// encodedForm.append("InKEY:-" + key);

// encodedForm.append("Indata:-" + dataLen);

int $\mathrm{a}=1$;

for (int $\mathrm{i}=0 ; \mathrm{i}<$ dataLen; $\mathrm{i}++$ ) \{

if (dataStrTrack $+2==$ dataLen $)\{$

partData $=$

Integer.parseInt(data.substring(dataStrTrack));

flag = true;

\} else if (dataStrTrack < dataLen) \{
keyStrTrack $=0$, resultData;

String resultStr = "", tmpStr = "';

boolean flag $=$ false;

int $\mathrm{a}=1$;

for (int $\mathrm{i}=0$; $\mathrm{i}<$ dataLen; $\mathrm{i}++$ ) \{

if (dataStrTrack $+2==$ dataLen) $\{$ partData $=$

Integer.parseInt(data.substring(dataStrTrack));

flag = true;

\} else if (dataStrTrack < dataLen) \{ partData

$=$ Integer.parseInt(data.substring(dataStrTrack,

dataStrTrack + 2)); \}

partKey $=$

Integer.parseInt(key.substring(keyStrTrack,

keyStrTrack + 2));

resultData = partData - partKey;

tmpStr = String.valueOf(resultData);

if (resultData $<=9)$ \{

tmpStr = "0" + tmpStr; $\}$

encodedForm.append("In" + partData + "+" +

partKey + "=" + tmpStr);

resultStr $+=$ tmpStr;

if (flag) \{

break;

keyStrTrack $=$ keyStrTrack +2 ;

dataStrTrack $=$ dataStrTrack +2 ;

if $($ keyStrTrack $>=$ keyLen/*6*/) \{

keyStrTrack $=0 ; \quad\} \quad\}$

int len = resultStr.length ()$, \operatorname{tmpA}, \operatorname{tmpB}$;

data $=$ resultStr;

dataStrTrack $=0$

String tmpres = "";

flag = false;

for (int $\mathrm{i}=0 ; \mathrm{i}<$ len; $\mathrm{i}++)\{$ 
if (dataStrTrack $+2==$ len) \{ partData $=$

Integer.parseInt(data.substring(dataStrTrack)); flag = true;

\} else if (dataStrTrack < len) \{ partData $=$

Integer.parseInt(data.substring(dataStrTrack, dataStrTrack + 2)); \} if (partData $>=1 \& \&$

partData $<=27)\{$ partData $+=64$;

tmpres $=$ tmpres $+($ char $)$ partData; $\}$ else \{ tmpres $=$ tmpres $+"$ "; $\quad\}$

dataStrTrack $+=2$

if (flag) \{ break; \} \}

rT1.setString(tmpres); \}

public void commandAction(Command c, Displayable d) \{

if $(\mathrm{c}==\mathrm{iC})\{$
initConvertedForm();

display.setCurrent(convertedForm);

convertData();

convertedKey () ;

\} else if $(\mathrm{c}==\mathrm{cC})\{$

initEncodedForm();

encodedData();

display.setCurrent(encodedForm);

\} else if $(\mathrm{c}==\mathrm{eC})\{$

initResultForm();

decodeData();

display.setCurrent(resultForm);

\} else if $(\mathrm{c}==\mathrm{rC})\{$

initInputForm();

display.setCurrent(inputForm);

\} else if ( $c==$ exit) \{

notifyDestroyed();

\}$\quad\}$

\section{M-COMMERCE BACKGROUND}

Encryption module is utilized for security purpose in websites and on mobile phone. Here security is estimated from customer or user and system side. The customer want to do the transaction of purchasing an item from a websites either using computer or mobile phone. Customer will purchase the Copan of worth Rs.200 from the market. The Copan contains number. Custer will scratch the number and removed the layer on it. The system administrator has inserted Copan number 111, 222, 333, 444 etc this numbers previously. The data base contains these numbers. The customer came with the same Copan number of above mention, he will be allowed for transaction. The Copan will be compared with database as in the figure no (3). Because the person has paid for it so he can enter in to the system for their transaction.

The inserted data would be encrypted and database will be implemented with the encrypted form as in the figure no 1 . The first page contains the inputs of the data like (1) Name (2) e-mail which are the real parameterized input of the encryption algorithm. On the basis of these data and (3) Copan Number collectively enter and user will enter in the website module. So the algorithm inputs are important for initiating the transaction.

\section{PRACTICAL USE OF ALGORITHM}

The encryption algorithm is encrypting the input text using encryption algorithm. The text is converting in not to understanding format. The encrypted text is stored in database as encrypted format.

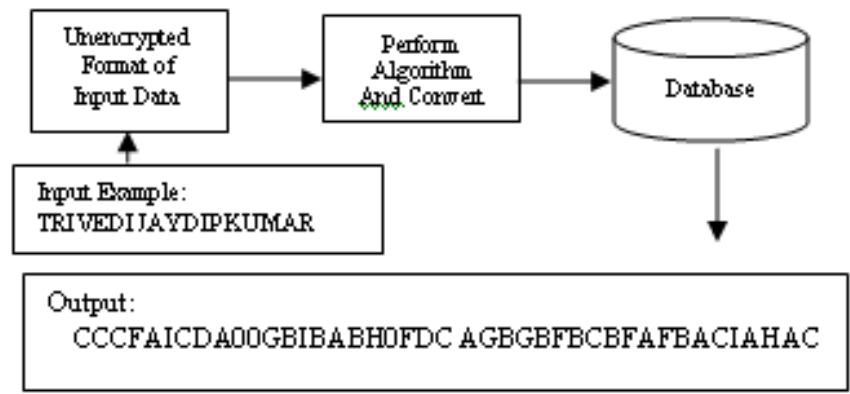

Figure 2: Input output format for database

Credit conformation initiating the process: The website of the encryption module's database contains the Copan number Exp 111,222,333,444,555, and the system administrator has put Copan in the market for customer for facilities the items like Example (1) Learning tutorial (2) Scholarly article (3) Application Program etc. Customer purchase Copan and scratch it for entering number into the website of the encryption module. The module compares the database what the customer enter the Copan number. If the Copan number found then he will be enter in to the website module for transection. 


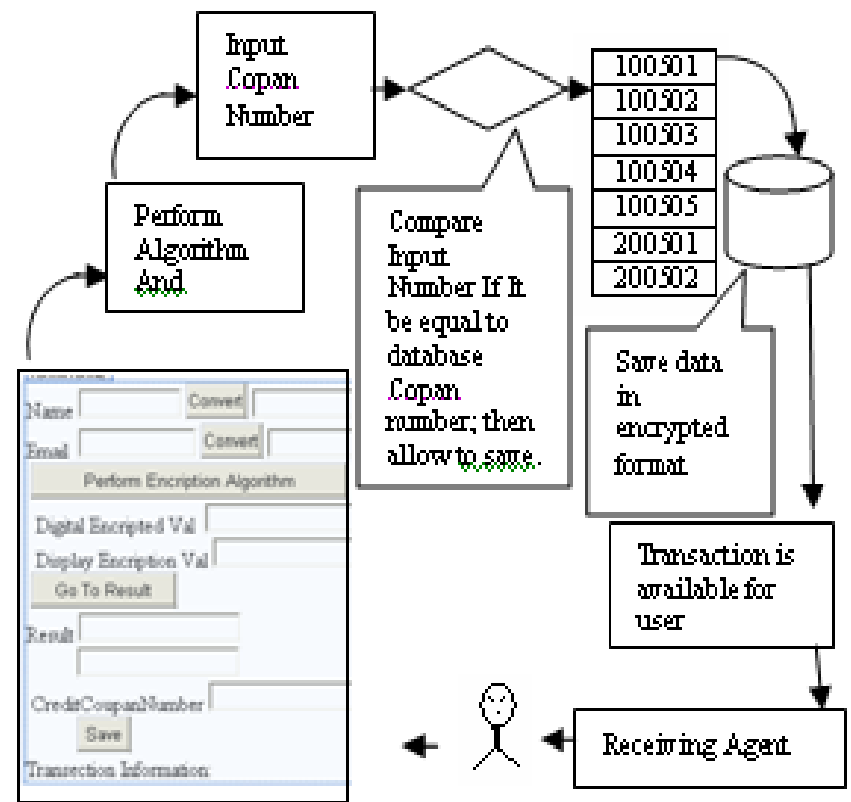

Figure 3, General Architecture for Data input, performance of algorithm, store the data and customer credit conformation.

\title{
IX. DATA AND ANALYSE
}

The parameterized data to be inserted in the simulator for encrypting the data for establishing transaction. And seems to be secure in the database from customer side as well as from system side.

1. Input Data:

\author{
Name: GANESH \\ E-mail: G@yahoo \\ Copan Number: 111
}

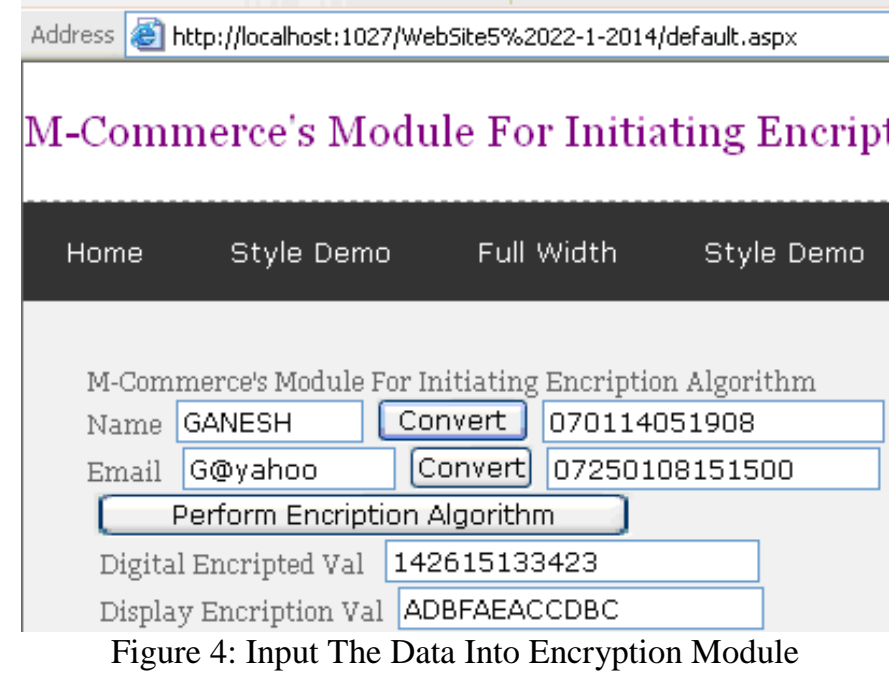

1. Out Put Data:

Name Encrypted Format: 142615133423

E-mail Encrypted Format: ADBFAEACCDBC 2.Input Data:

Out put can be seen in the figure 7: Encrypted format.

Name: SARASWATI

E-mail: s@gmail 


\section{M-Commerce's Module For Initiating Encrip-}

\begin{tabular}{|c|c|c|c|c|}
\hline & Home & Style Demo & Full $\mathrm{n}$ & Style Demo \\
\hline & \multicolumn{4}{|c|}{ M-Commerce's Module For Initiating Encription Algorithm } \\
\hline & Name & SARASWATI & Convert & 190118011923012009 \\
\hline & Email & s@gmail & Convert & 19071301091200 \\
\hline & \multicolumn{4}{|c|}{ Perform Encription Algorithm } \\
\hline & \multicolumn{4}{|c|}{ Digital Encripted Val 380831022835013916} \\
\hline Copan Number:222 & \multicolumn{4}{|c|}{ Display Encription Val CHOHCAOBBHCEOACIAF } \\
\hline
\end{tabular}

Figure 5: Input the Data Into Encryption Module

\section{Out Put Data:}

Name Encrypted Format: 380831022835013916

E-mail Encrypted Format: CHOCHA0BBHCE0ACIAF

Out put can be seen in the figure 7: Encrypted format.

\section{Input Data:}

Name: SANGITABEN

E-mail: sht@dd

Copan Number: 333

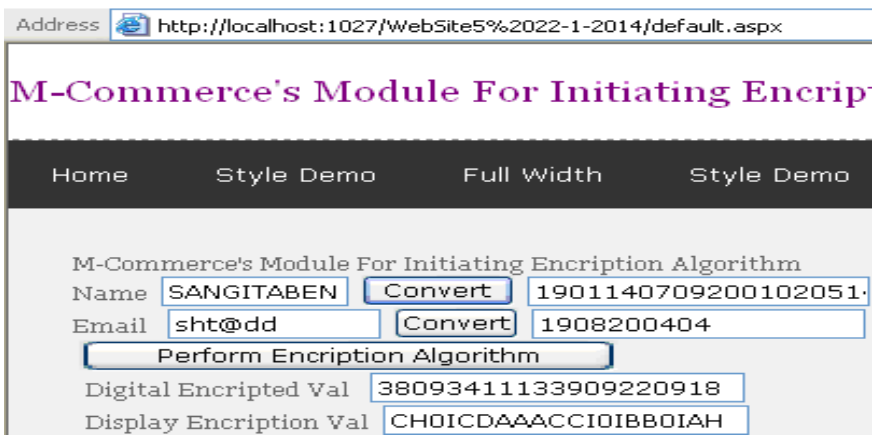

Figure 7: Input The Data Into Encryption Module

\section{Out Put Data:}

Name Encrypted Format:

E-mail Encrypted Format:

Out put can be seen in the figure 7: Encrypted format.

\section{X. $\quad$ RESULT AND TEST THE HYPOTHESIS}

Encrypted format of the inputs in the module could not be understood by the customer from the customer side as well as system administrator from system side. Here, the under mention (figure 7) database instruction of encryption module proves the result. The database table1 contain encryption module and Name Column, E-mail Column contain encrypted format of the data.

\begin{tabular}{|c|c|c|c|c|c|}
\hline \multicolumn{6}{|c|}{ WebSite5 22-1-2014 - Microsoft Visual Studio } \\
\hline \multicolumn{6}{|c|}{ 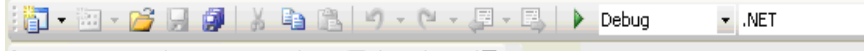 } \\
\hline \multicolumn{6}{|c|}{ 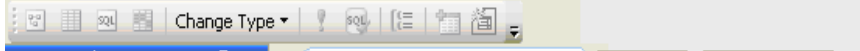 } \\
\hline \multicolumn{3}{|l|}{ Server Explorer } & \multicolumn{3}{|l|}{ Start Page } \\
\hline 와 $\times 1$, tै & & Name & \multicolumn{2}{|l|}{ Email } & Cre \\
\hline \multirow{6}{*}{ 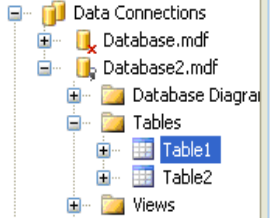 } & \multirow[t]{5}{*}{1} & 142615133423 & \multicolumn{2}{|c|}{ ADBFAEACCDBC } & 111 \\
\hline & & 380831022835013916 & \multicolumn{2}{|c|}{ CHOHCAOBBHCEOACIAF } & 222 \\
\hline & & 38093411133909220918 & \multicolumn{2}{|c|}{ CHOICDAAACCIOIBBOIAH } & 333 \\
\hline & & 1622171409260524092313 & \multicolumn{2}{|c|}{ AFBBAGADOIBF0EBDOIBCAC } & 444 \\
\hline & & 3619171427342205202625 & \multicolumn{2}{|c|}{ CFAIAGADBGCDBBOEBOBFBE } & 555 \\
\hline & * & $\operatorname{mul}$ & \multicolumn{2}{|l|}{ nur } & nut \\
\hline
\end{tabular}

Figure 7: Encrypted format in database of encryption module in the website 


\section{Crucial Performance of Algorithms:}

- Data entry can be performed at Input 1,2 and 3 using encryption algorithm is impotent matter What the work is indicating in the Figure No 4,5 and 6.

- Encryption algorithm is helping for convert the data in to Encrypted format is essential for database. What the figure

7 is indicating.

\section{CONCLUSION}

Thus the work indicated the instruction of "Applying encryption algorithm practically on m-commerce background" and at the end obtained security and crucial performance.

\section{REFERENCES}

[1] Trivedi J H, Trivedi P H, Thakker S T, Makwana M N, Automatic and Semi Automatic Transaction of roadcasting and It's Receiving Agent Based M- commerce Business Model

[2] Trivedi J H, Trivedi P H, Darji J H, Patel H D, Designing and prototyping encryption algorithms: Working as secure $\mathrm{M}$-commerce transaction of broadcasting and its receiving agent based m-commerce model, IOSRJEN,Vol.3,Issue 8, pp.25-33 (2013) ISSN: 2250- 3021

[3] Pavan Soni, M-Payment Between Banks Using SMS,2010 IEEE Vol.98,No.6, June 2010 